\title{
Interdependent Components of Web Accessibility
}

\author{
Wendy A. Chisholm \\ World Wide Web Consortium (W3C) \\ Web Accessibility Initiative (WAI) \\ Massachusetts Institute of Technology (MIT), Computer \\ Science and Artificial Intelligence Laboratory (CSAIL) \\ 32 Vassar Street \\ Cambridge, MA 02319-4307, USA \\ $+1.617 .395 .4103$ \\ wendy@w3.org
}

\author{
Shawn Lawton Henry \\ World Wide Web Consortium (W3C) \\ Web Accessibility Initiative (WAI) \\ Massachusetts Institute of Technology (MIT), Computer \\ Science and Artificial Intelligence Laboratory (CSAIL) \\ 32 Vassar Street \\ Cambridge, MA 02319-4307, USA \\ $+1.617 .395 .7664$ \\ shawn@w3.org
}

\begin{abstract}
Increasingly, the Web is providing unprecedented access to information and interaction for people with disabilities. However, the Web will not be equally accessible, allowing people with disabilities to access and contribute to the Web, until:

- Authoring tools and development environments (including content managements systems such as blogging applications) produce accessible Web content and have accessible interfaces;

- Browsers, multimedia players and assistive technologies provide a completely usable and accessible experience;

- Content is designed to be accessible.

Web accessibility relies on tools that are designed to work together and support the needs of the people who use them. This paper describes how Web accessibility depends on several components working together. It demonstrates the relationship between the World Wide Web Consortium (W3C) Web Accessibility Initiative (WAI) guidelines: Web Content Accessibility Guidelines (WCAG), Authoring Tool Accessibility Guidelines (ATAG), and User Agent Accessibility Guidelines (UAAG).
\end{abstract}

\section{Categories and Subject Descriptors}

H.1.2 User/Machine Systems: Human Factors

\section{General Terms}

Management, Documentation, Design, Human Factors, Standardization.

\section{Keywords}

Permission to make digital or hard copies of all or part of this work for personal or classroom use is granted without fee provided that copies are not made or distributed for profit or commercial advantage and that copies bear this notice and the full citation on the first page. To copy otherwise, or republish, to post on servers or to redistribute to lists, requires prior specific permission and/or a fee.

W4A at WWW2005, 10th May 2005, Chiba, Japan

Copyright 2005 ACM 1-59593-036-1/05/05 ...\$5.00 disabilities, accessibility, authoring tools, user agents, Web content, guidelines, technical specifications, blog, assistive technology, WCAG, ATAG, UAAG, W3C, WAI

\section{INTRODUCTION}

Increasingly, the Web is providing unprecedented access to information and interaction for people with disabilities. However, most Web sites still have accessibility barriers that make it difficult or impossible for many people with disabilities to use the site. And most Web tools are not fully accessible to people with disabilities, making it difficult or impossible for them to contribute Web content. However, if Web sites and Web tools were made accessible, people with disabilities could use the Web effectively. [1]

Much of the focus of Web accessibility in the past has been on the responsibilities of Web content producers. This view misses the crucial interdependence of several components working together, including:

- technical components:

- technical specifications,

- content,

- tools;

- human components:

- content producers,

- end-users,

- tool developers.

\subsection{People with Disabilities as Content Producers}

The World Wide Web was initially designed to be a medium for sharing information, where people not only read information, they also contribute information.

The dream behind the Web is of a common information space in which we communicate by sharing information. - Tim Berners-Lee [2] 
To realize this dream for people with disabilities, the tools used to create and modify Web content must also be accessible. Tool developers have often mistakenly assumed that people with disabilities are unable to create Web content. However, consider the following examples:

- People have a wide range of visual abilities and disabilities from blurred vision, to partial sight, to complete blindness which often change over time. Many visual artists, including masters such as Monet and Degas, continue to work even after their sight diminishes either from aging-related changes in the eye or from disease or injury [3].

- Most design applications include keyboard equivalents to make very fine movements, movements that even the most deft illustrator may not be able to accomplish via a mouse or stylus. A designer who does not have fine motor control because of disability could use keyboard equivalents instead of a mouse or stylus. Thus, a person who cannot even hold a pencil could be an accomplished graphic artist.

- The Web has the potential to provide a unique communication vehicle for people with certain cognitive or intellectual disabilities who may have difficulty communicating with written language. Programs allow people to develop content using images, audio, and video.

Consider also that content is often produced by a team of people with differing skills and abilities. Team members may use different features of the same tool or may use different tools. As team members age, their needs and uses of familiar tools may change.

Ensuring that people with disabilities are able to contribute content is an important aspect of making the Web accessible.

\section{DESCRIPTION OF COMPONENTS}

\subsection{Technical Components}

Technical specifications describe the features of languages that are used to create and deliver Web content. W3C specifications are intended to be "compatible with one another and allow any hardware and software used to access the Web to work together." [4] Technical specifications must be designed with accessibility in mind: otherwise, the technology might not support necessary accessibility features.

Content is the information that forms Web sites and Web applications: the code and markup that define the structure, presentation, and interaction, as well as text, images, and sounds that convey information to the end-user.

User agents are tools used by end-users to view and interact with content including Web browsers, media players, and assistive technologies.

Assistive technologies include hardware and software that may be used in addition to a browser or media player such as screen readers, alternative keyboards, switches, and scanning software.

Authoring tools include any software or service that developers use to create or modify Web content. This includes what-you-seeis-what-you-get (WYSIWYG) tools specifically designed to produce Web content, word processor tools that transform documents into Web formats, and content management systems that generate Web content. Blogging applications are another type of authoring tool.

The blogging phenomenon is a natural extension of the Web paradigm whereby everyone is potentially both a content user and a content provider. Even more than Web design software and personal home pages, blogs make it easy for anyone to publish their thoughts, opinions, feelings, experiences, and knowledge on the Internet. This ease of publication, coupled with increasing media coverage, has grown into a revolution in how the Web is being used. - Kynn Bartlett [5]

Evaluation tools are used to check Web content for validation and accessibility errors. Evaluation tools can identify syntax errors and make the evaluation process more efficient; however, human judgment is required to determine if content is accessible. Using an evaluation tool is similar to using a spell checker to look for syntax errors.

\begin{abstract}
A spell checker can identify words that are not in its dictionary, and it can suggest similar words to fix the potentially mis-spelled word. A human must then decide if the word is mis-spelled. Another issue is that spell checkers will not identify wrong words that are properly spelled. For example, if I type, "good text tool" when I meant to type, "good test tool" the spell checker will not identify it as a spelling error. - Shawn Lawton Henry [6]
\end{abstract}

\subsection{Human Components}

End-users are the people using the Web content and user agents. End-users have differing experiences and abilities. Some configure their tools, and configurations change with different devices in different situations. In some cases, they will use adaptive strategies to interact with content. For example, someone who cannot visually skim through content to get an overview of a Web page may use their tools to read out links, headers, list items, or other structural items in Web content.

Content producers are the people who design, code, edit, and otherwise create Web content.

Tool developers design, code, and test the user agents, authoring tools, assistive technologies, and evaluation tools used by content producers and end-users to create, edit, evaluate, and interact with Web content.

One individual can fill multiple roles. As described in the "People with disabilities as content producers" section of the introduction, people should be able to contribute Web content (be "content producers”) as well as access Web content (as “end-users”). 


\section{RELATIONSHIPS BETWEEN TECHNICAL COMPONENTS AND HUMAN COMPONENTS}

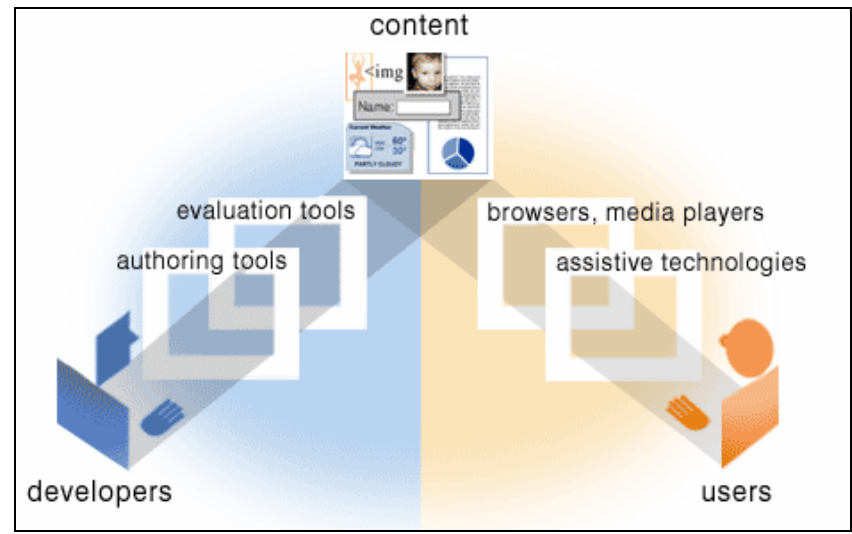

Figure 1: Components of Web Accessibility [7]

Figure 1 illustrates the relationship between the basic components of Web accessibility. Content producers ("developers" in the figures) usually use authoring tools and evaluation tools to create Web content. End-users use Web browsers, media players, assistive technologies, or other user agents to get and interact with the content. Tool developers provide features and functionality in their authoring tools, evaluation tools, and user agents based on user needs.

\subsection{Interdependencies between Components}

To illustrate how components must work together in order for the Web to be accessible, consider providing and using alternative text descriptions for images as an example.

Technical specifications provide the framework to create content. If a technical specification does not provide a rich enough vocabulary, then the content can not be accessible. For example, if a specification does not provide a way to describe relationships between content, then the content producer is either unable to provide that information or must provide it in a non-standard way.

An example where a specification provides a rich enough vocabulary is the HTML 4.01 definition of the img element. The img element is used to embed images into content and the alt attribute enables content producers to provide a text alternative description of the image.

\section{alt}

For user agents that cannot display images, forms, or applets, this attribute specifies alternate text. The language of the alternate text is specified by the lang attribute.

Specifying alternate text assists users without graphic display terminals, users whose browsers don't support forms, visually impaired users, those who use speech synthesizers, those who have configured their graphical user agents not to display images, etc.
The alt attribute must be specified for the IMG and AREA elements. [8]

The other technical components have the following responsibilities with respect to providing alternative text via the alt attribute:

- Authoring tools enable, facilitate, and promote providing alternative text. The interface to insert an image and provide alternative text should be accessible

- Evaluation tools check that alternative text exists and helps determine if it is appropriate. "Appropriate" means the text alternative conveys the same information, provides the same function, is marked such that it can be ignored, or in cases where text is not able to convey the same information or provide the same function, such as artistic works, alternative text provides a label or brief description of the content. [9]

- User agents provide human and machine interfaces to the alternative text.

- Assistive technologies provide a human interface to the alternative text in various modalities.

The WAI guidelines, which are described in the "Bring Together the Components" section, define how to implement alternative text for accessibility in the different technical components.

The human components have the following responsibilities to provide and access alternative text as follows:

- Content producers use authoring tools and evaluation tools to provide the appropriate alternative text.

- End-users know how to get the alternative text from their user agent and assistive technologies as needed.

\subsection{Getting Accessibility into the Implementation Cycle}

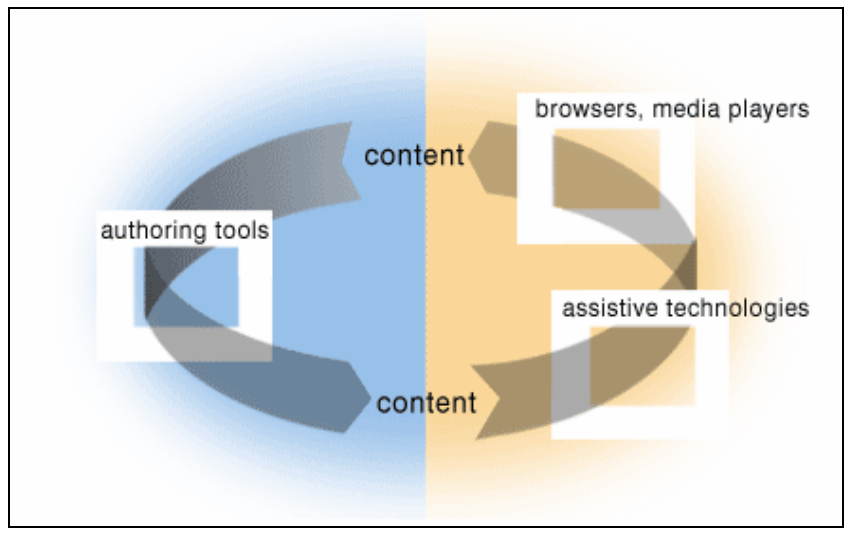

Figure 2: Cycle of Accessibility Implementation [7]

The interdependencies between components have historically fed a "chicken and egg" problem of who goes first in implementing an accessibility feature. Figure 2 illustrates this implementation cycle without a clear beginning. Content producers have been reluctant to implement a new feature from a technical specification until user agents support the feature or authoring tools make a feature easy to implement. Meanwhile, user agent developers often wait 
for a critical mass of content producers to use a new feature before they incorporate it into the next release of a user agent. The result is that often accessibility features are not implemented or they are implemented differently between or within components.

When accessibility features are effectively implemented in one component, the other components are more likely to implement them. However, if an accessibility feature is not implemented in one component, there is little motivation for the other components to implement it when it does not result in a more accessible user experience. For example:

- If an authoring tool does not enable, facilitate, or promote providing alt for images, content producers may not provide text descriptions and then end-users may be unable to use content.

- If alternative text is provided in the content but the user agent does not provide the human interface to the alternative text, then end-users may be unable to use the content.

\subsection{Compensating for Lack of Support}

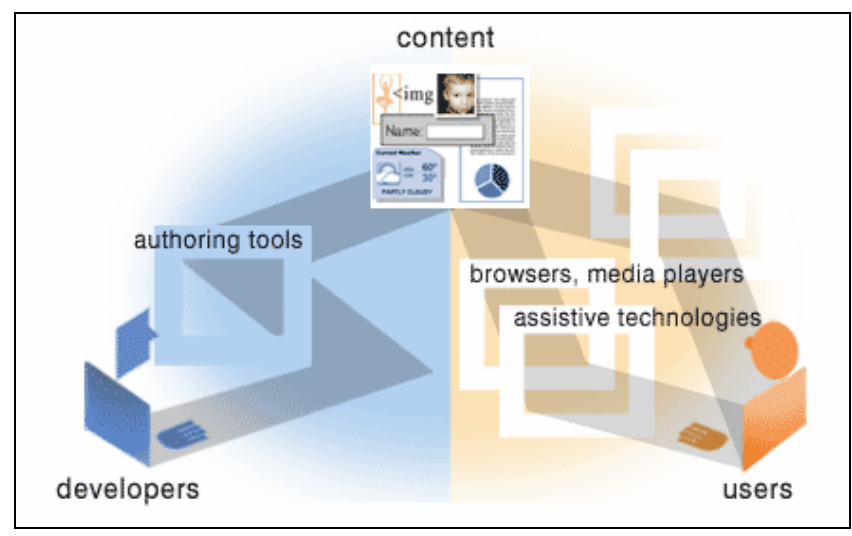

Figure 3: Compensating for Lack of Accessibility Support [7]

If one technical component has poor accessibility support, sometimes other components can compensate through "workarounds" that often require extra effort both initially to create and long-term to maintain. Figure 3 illustrates the compensation for lack of accessibility support in tools, as described below.

When authoring tools and user agents do not support an accessibility feature, sometimes the content producer can compensate with work-arounds. For example, instead of relying on an authoring tool to produce accessible markup, the content producer may write the markup "by hand"; or, when a user agent doesn't provide the ability to navigate through the structure of the content efficiently, the content producer may provide additional links.

In some cases, the end-user can compensate for lack of accessibility support in browsers or content. For example, endusers may use combinations of user agents or assistive technologies or rely on search or other features of the browser. Unfortunately, for many accessibility issues, there is no workaround for the end-user and the content is inaccessible.

\section{BRINGING TOGETHER THE COMPONENTS}

The Web Accessibility Initiative (WAI) [10] of the World Wide Web Consortium (W3C) [11] helps coordinate international Web accessibility efforts to bring together the technical and human component considerations. WAI works with:

- W3C Working Groups to produce technical specifications that support accessibility;

- User agent, authoring tool, and evaluation tool developers to create WAI guidelines for implementation of W3C specifications, including:

- Authoring Tool Accessibility Guidelines (ATAG),

- User Agent Accessibility Guidelines (UAAG);

- Content producers to create WAI guidelines for using W3C specifications to create accessible Web content, including:

- Web Content Accessibility Guidelines (WCAG);

- End-users to understand issues and advocate for aspects of accessibility in each of the other components.

By coordinating these activities within the W3C, WAI is uniquely positioned to effect Web accessibility at the beginning of the Technical Specification development process instead of retrofitting afterwards. The WAI guidelines help create a blueprint showing how these components can work well together. This also helps reduce the "chicken and egg" issue that arises from different parties waiting for someone else to 'go first' by encouraging them all to act at the same time.

Figure 4 illustrates how WAI's work fits into the components of Web Accessibility.

\subsection{Authoring Tool Accessibility Guidelines}

Authoring Tool Accessibility Guidelines 1.0 (ATAG 1.0) [12] was published as a W3C Recommendation in February 2000. ATAG 1.0 provides guidelines for authoring tools to produce accessible content via an accessible authoring interface. Through prompts, alerts, templates, and other features, authoring tools can enable, encourage, and assist content producers to create accessible Web content. As described in the "People with disabilities as content producers" section of the introduction, people must also be able to contribute content. Therefore, the authoring tools themselves must be accessible. ATAG 2.0 [13] is currently under development to clarify minor issues with ATAG 1.0 and to update references to other guidelines.

While ATAG is primarily written for authoring tool developers, ATAG and supporting documents are also intended to meet the needs of many different audiences, including policy makers, managers, and others. For example:

- People who want to choose authoring tools that are more accessible can use ATAG to evaluate authoring tools [14] 


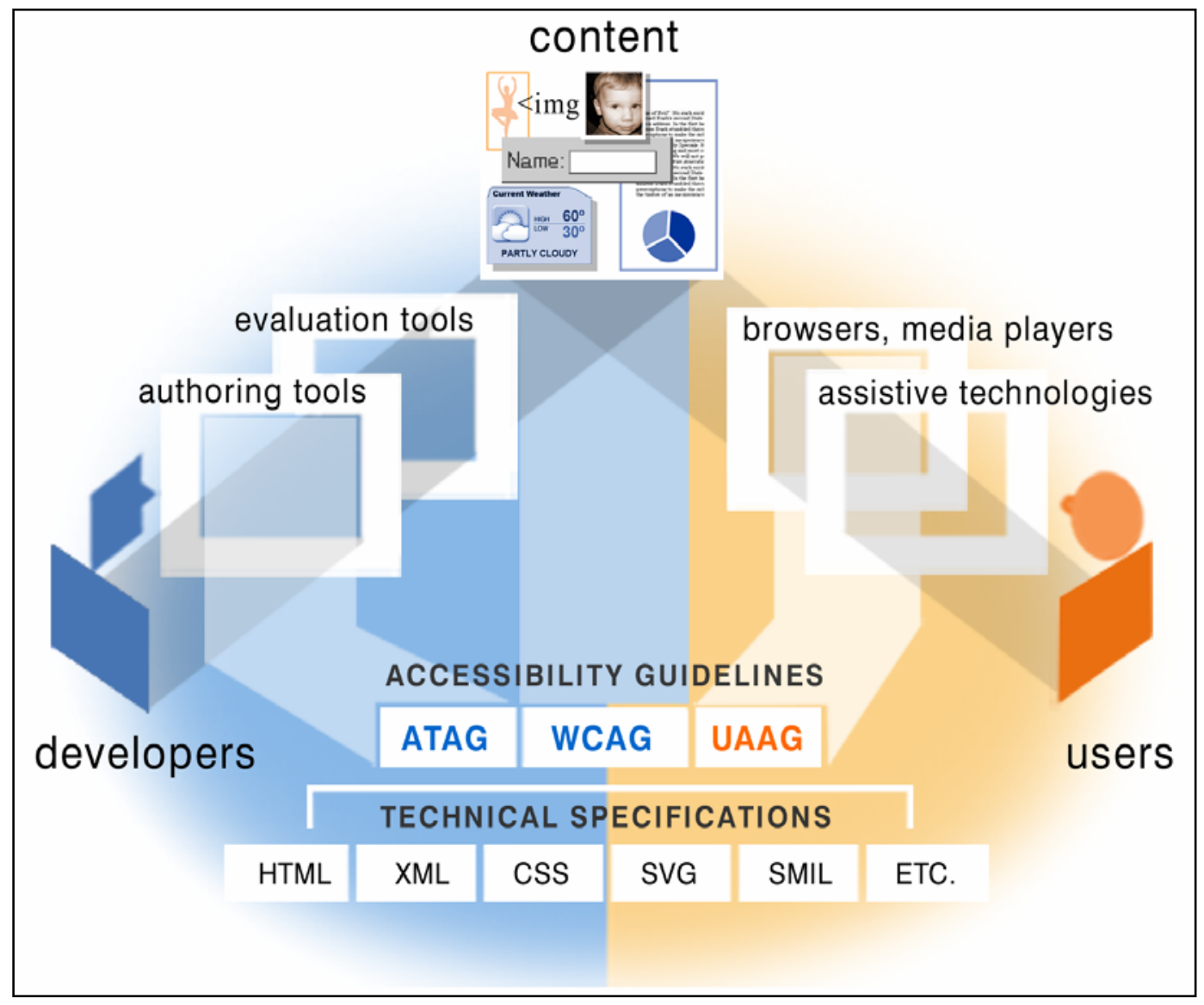

Figure 4: Components and Guidelines for Web Accessibility [7]

- People who want to encourage their existing authoring tool developer to improve accessibility in future versions can refer the authoring tool vendor to ATAG

\subsection{Web Content Accessibility Guidelines}

Web Content Accessibility Guidelines 1.0 (WCAG 1.0) [15] was published as a W3C Recommendation in May 1999. WCAG 1.0 explains how to make Web content accessible to people with disabilities. While WCAG is targeted primarily for content producers, it is also used by others: authoring tool developers use it to create tools that generate accessible content, user agent developers use it to create tools that utilize accessible content, and evaluation tool developers use it to create tools that discover accessibility issues with content.

Since WCAG 1.0 was published, many organizations have adopted or referenced WCAG 1.0 in their policies. Feedback on WCAG 1.0 from developers and policy makers forms the basis of the requirements for WCAG 2.0 [16]. To satisfy these requirements, WCAG 2.0 differs from WCAG 1.0 by introducing a new priority scheme; defining testable success criteria; removing technology-specific information from the guidelines; providing testable information in checklists, techniques, and test suites; and providing an overview of materials to orient users to the suite of documents.

\subsection{User Agent Accessibility Guidelines}

User Agent Accessibility Guidelines 1.0 (UAAG 1.0) [17] was published as a W3C Recommendation in December 2002. The UAAG 1.0 documents explain how to make user agents accessible to people with disabilities, particularly to increase accessibility to Web content. UAAG 1.0 is primarily for developers of Web browsers, media players, assistive technologies, and other user agents.

UAAG and supporting documents are also intended to meet the needs of many different audiences, including policy makers, managers, and others. For example:

- People who want to choose user agents that are more accessible can use UAAG to evaluate user agents

- People who want to encourage their existing user agent developer to improve accessibility in future versions can refer the user agent vendor to UAAG

\subsection{W3C Technical Specifications}

The mission of the Protocols and Formats Working Group (PFWG) [18] is to increase the support for accessibility in Web Technical Specifications. This supports the W3C mission of promoting universal access and interoperability across the Web. The PFWG coordinates across W3C Working Groups to improve 
the level of accessibility support in Web technology as it evolves and is implemented. The PFWG works to ensure that:

- Web content and applications implemented using the specified technologies can be adapted for people with disabilities with a high level of usability,

- Web technologies interoperate well with assistive technologies,

- Accessibility features are integrated into and a natural part of technical specifications not requiring a high level of additional effort by content producers nor bloating content

\section{CONCLUSION}

While this paper focuses on accessibility, the relationship between the components is an important aspect of Web development overall. Lack of cooperation between the components creates problems in addition to accessibility. For example, because of the inconsistencies between user agents, content producers have bridged the gap with work-arounds for content to work across browsers. This extra effort has added "at least $25 \%$ to the cost of developing all sites." [19]

It is essential that the technical and human components of Web development and interaction work together in order for the Web to be accessible to people with disabilities. This paper discusses the interdependent relationship between the components and how improvements in specific components could substantially improve Web accessibility. WAI guidelines address each of the technical components. The W3C and other organizations are working with developers, content producers, specification writers, and endusers to realize the dream of the Web as an information space where everyone, regardless of ability, is able to share information.

\section{ACKNOWLEDGMENTS}

The authors would like to thank the participants in W3C WAI Working Groups who have developed materials referenced in this article, including participants of the Web Content Accessibility Guidelines Working Group, Authoring Tool Accessibility Guidelines Working Group, Protocols and Formats Working Group, and Education and Outreach Working Group.

\section{RESOURCES}

The following resources provide additional information directly related to this paper. Other resources are listed in the References section below.

- Essential Components of Web Accessibility [7] - a shorter, more simple coverage of the issues addressed in this paper

- How People with Disabilities Use the Web [20]

- Authoring Tool Accessibility Guidelines (ATAG) Overview [21]

- Web Content Accessibility Guidelines (WCAG) Overview [22]

- User Agent Accessibility Guidelines (UAAG) Overview [23]

- Evaluating Web Sites for Accessibility [24]
- Evaluation, Repair, and Transformation Tools for Web Content Accessibility [25]

\section{REFERENCES}

[1] Henry, S.L., ed. "Introduction to Web Accessibility." http://www.w3.org/WAI/intro/accessibility

[2] Berners-Lee, T. "The World Wide Web: A very short personal history." http://www.w3.org/People/BernersLee/ShortHistory

[3] RNIB, "Painting from a New Perspective: painting and sight loss." http://info.rnib.org.uk/blindartists

[4] Jacobs, I. ed. "About the World Wide Web Consortium (W3C)." http://www.w3.org/Consortium/

[5] Bartlett, K. "Accessibility of the Blogging Revolution," Center On Disabilities: Technology And Persons With Disabilities Conference 2004, Los Angeles, CA (2004) http://www.csun.edu/cod/conf/2004/proceedings/150.htm

[6] Henry, S.L. "Web Accessibility Evaluation Tools Need People." Hhttp://www.uiaccess.com/evaltools.htmlH

[7] Henry, S.L. ed. "Essential Components of Web Accessibility."

Hhttp://www.w3.org/WAI/intro/componentsH

[8] Raggett, D; Le Hors, A; Jacobs, I. eds. "HTML 4.01 Specification: How to specify alternate text" W3C Recommendation (1999). http://www.w3.org/TR/html4/struct/objects.html\#adef-alt

[9] Caldwell, B., et al. eds. "Web Content Accessibility Guidelines 2.0." W3C Working Draft (2004). http://www.w3.org/TR/2004/WD-WCAG20-20041119/

[10] Web Accessibility Initiative. http://www.w3.org/WAI/

[11] World Wide Web Consortium. http://www.w3.org/

[12] Treviranus, T., et al. eds. "Authoring Tool Accessibility Guidelines 1.0." W3C Recommendation (2000) http://www.w3.org/TR/2000/REC-ATAG10-20000203

[13] Treviranus, T., et al.eds. "Authoring Tool Accessibility Guidelines 2.0." W3C Working Draft (2004) http://www.w3.org/TR/2004/WD-ATAG20-20041122/

[14] Brewer, J. ed. "Selecting and Using Authoring Tools for Web Accessibility." http://www.w3.org/WAI/impl/software.html

[15] Chisholm, W.; Vanderheiden, G.; Jacobs, I. eds. "Web Content Accessibility Guidelines 1.0." W3C Recommendation (1999) http://www.w3.org/TR/1999/WAIWEBCONTENT-19990505 
[16] Vanderheiden, G.; Chisholm, W. eds. "Requirements for WCAG 2.0" W3C Working Draft (2002)

http://www.w3.org/TR/2002/WD-wcag2-req-20020426

[17] Jacobs, I.; Gunderson, J.; Hansen, E. eds. "User Agent Accessibility Guidelines 1.0." W3C Recommendation (2002) http://www.w3.org/TR/2002/REC-UAAG10-20021217/

[18] Protocols and Formats Working Group. http://www.w3.org/WAI/PF/

[19] Web Standards Project. "WASP: Fighting for Standards." http://www.webstandards.org/about/

[20] Brewer, J.ed. " How People with Disabilities Use the Web." http://www.w3.org/WAI/EO/Drafts/PWD-Use-Web/

[21] Henry, S.L.; May, M. eds. "Authoring Tool Accessibility Guidelines (ATAG) Overview." http://www.w3.org/WAI/intro/atag.html

[22] Henry, S.L. ed. "Web Content Accessibility Guidelines (WCAG) Overview."

http://www.w3.org/WAI/intro/wcag.html

[23] Henry, S.L.; May, M. eds. "User Agent Accessibility Guidelines (UAAG) Overview." Hhttp://www.w3.org/WAI/intro/uaag.htmlH

[24] Brewer, J.; Letourneau, C. eds. "Evaluating Web Sites for Accessibility.” http://www.w3.org/WAI/eval/

[25] Abou-Zahra, S.; Chisholm, W.; Brewer, J. eds. "Evaluation, Repair, and Transformation Tools for Web Content Accessibility."

Hhttp://www.w3.org/WAI/ER/existingtools.htmlH 